\title{
Three-Compartment Model of CAR T-cell Immunotherapy
}

\author{
Brendon de Jesus Rodrigues - Luciana R. Carvalho Barros - Regina C. \\ Almeida
}

Received: date / Accepted: date

\begin{abstract}
Immunotherapy has gained great momentum with chimeric antigen receptor $\mathrm{T}$-cell $(\mathrm{CAR} \mathrm{T})$ therapy, in which patient's $\mathrm{T}$ lymphocytes are genetically manipulated to recognize tumor-specific antigens to increase tumor elimination efficiency. Improved CAR $\mathrm{T}$ cell immunotherapy requires a better understanding of the interplay between CAR T cell doses and tumor burden, administration protocol, toxicity, resistance to immunotherapy, among other features. We developed a three-compartment mathematical model to describe tumor response to CAR T cell immunotherapy in immunodeficient mouse models. It encompasses interactions between tumor cells, effector and long-term memory CAR T cells such as tumor induced immunosuppression effects, conversion of memory $\mathrm{T}$ cells into effector $\mathrm{T}$ cells in the presence of tumor cells, and individual specificities considered as uncertainties in the parameters of the model. The model was able to represent two different immunotherapy scenarios with different CAR receptors and tumor targets reported in the literature. Further in silico studies considering different dosing quantities and tumor burden showed that the proposed model can represent the three possible therapy outcomes: tumor elimination, equilibrium, and escape. We found that therapy effectiveness may also depend on small variations in the parameter values, regarded as intrinsic individual specificities, as $\mathrm{T}$ cell proliferation capacity, as well as immunosuppressive tumor microenvironment factors. These issues may significantly
\end{abstract}

Brendon de Jesus Rodrigues · Regina C. Almeida

Laboratório Nacional de Computação Científica

Luciana R. Carvalho Barros

Instituto do Câncer do Estado de São Paulo

E-mail: lucianalpt@gmail.com reduce the chance of tumor elimination. In this way, the developed model provides potential use for assessing different CAR $\mathrm{T}$ cell protocols and associated efficacy without further in vivo experiments.

Keywords Mathematical Model . CAR T lymphocytes - Memory CAR T cells · Long-term Immunity · Tumor Induced Immunosupression

\section{Introduction}

Adoptive cell therapies have been considered a major advance in the fight against cancer, especially those associated with the hematopoietic system 27. CAR T cell immunotherapy is an adoptive cellular therapy in which $\mathrm{T}$ lymphocytes are taken from the blood of a patient, genetically modified to recognize antigens expressed by the patient's tumor, submitted to in vitro expansion and reinjected into the patient. Insertion of the CAR gene into $\mathrm{T}$ lymphocytes bestows the ability to recognize and directly attack tumor cells regardless of human leukocyte antigen presentation 32 . In 2017, the FDA approved the commercialization of two therapies with CAR T cells for the treatment of $\mathrm{CD} 19^{+} \mathrm{B}$ cells malignancies [16, 26. Other target proteins have been studied recently, such as CD123 that is expressed in many hematological malignancies, including acute myeloid leukemia, Hodgkin's lymphoma, acute lymphoblastic leukemia, among others, which makes it a potential antineoplastic target 6 . Therapy with CAR T cells has been successful in eliminating or relieving endurable types of lymphomas and leukemia. Recent experimental studies have investigated the relationship between immunotherapy with CAR $\mathrm{T}$ cells and the development of immunological memory in cancer 30,5 . Using immunodeficient mouse models, 30 showed that CAR T 
bioRxiv preprint doi: https://doi.org/10.1101/779793; this version posted April 18, 2020. The copyright holder for this preprint (which was

123 therapy can eliminate Hodgkin's lymphoma and provide long-term immunity against recurrence of the same tumor. Immuno checkpoint blockade associated with CAR T cell therapy is also under investigation in models where CAR T cell therapy fail. Also using immunodeficient mouse models, 29 showed that tumor expressing indoleamine 2,3-dioxygenase (IDO) activity, an intracellular enzyme that has an inhibitory activity on $\mathrm{T}$ cells, can be better controlled by combining the CAR therapy with 1-methyl-tryptophan (1-MT), an IDO inhibitor. By the end of 2016, four different immune checkpoint blockade drugs were also approved for the treatment of lymphoma, melanoma, among other cancers. Current and future advances in the engineering of CAR and new immunologic checkpoint inhibitor drugs offer promising perspectives in the treatment of cancer [16].

Although the success of this type of therapy against hematologic cancers is promising, the mechanisms associated with failures have been reported and are subjects of recent investigations 19. Notably, many challenges remain to be addressed to improve response rates such as minimum effective $\mathrm{T}$ cell dose, subtypes of CAR T cells selection, adverse effects, combination with other types of therapy, suppressive microenvironment, and patient specificity, among others. Mathematical models may contribute to the understanding of these mechanisms [5, 2, 22, 23, confronting hypotheses and testing different settings 20, 39, 24. To this end, here we focus on the development of a mathematical model to describe the treatment of hematologic malignancy in immunodeficient mouse models with a CAR T immunotherapy and the formation of the immune memory pool. We calibrated the model parameters with in vitro and in vivo data presented in [30] for CAR T 123 therapy against Hodgkin's lymphoma. We then used the model to retrieve a different immunotherapeutic CAR scenario, using data from 29] for CAR T 19 therapy on Raji tumors. We complemented the in silico study investigating different therapy outcomes depending on the relationship between the tumor burden and CAR T cell number; the therapy effectiveness due to inhibition by immunosuppressive tumor microenvironments, and intrinsic individual specificities represented by the uncertainties in the parameters of the model. Our model simulations provide insights on critical mechanisms of CAR T-cell therapy, and show potential use for assessing different CAR $\mathrm{T}$ cell protocols and associated efficacy, complementing and potentially avoiding further in vivo experiments.

\section{Materials and methods}

In vitro and in vivo data

We used in vitro and in vivo data published in [30] and [29. The cytotoxic activity of CAR T cells was evaluated in a standard in vitro 4 -hour chromium-51 release assay [13. Bioluminescence imaging (BLI) data were used to track tumor growth time-course. We considered one BLI unit as one cell. Although we did not find any correspondence in the literature to convert BLI to cell number, BLI and total tumor cell number is directly correlated with the total number of cells as shown in 1. All used data were extracted from those sources using the free software [11] and, for completitude, are presented in the Supplementary Material.

\section{Model development}

In this work, we focus on the development of a threecompartment mathematical model, using ordinary differential equations, to describe the interactions between populations of a tumor, effector CAR $\mathrm{T}$ and memory CAR T cells. As we are dealing with immunodeficient mice, we consider that the effector CAR T cells come only from the immunotherapy, represented by populations of (activated) CAR T lymphocytes that we denote by $C_{T}$. The population of memory CAR T lymphocytes is denoted by $C_{M}$ and the tumor population is denoted by $T$. The change of each cell population in time depends on the balance among all factors contributing to its increase and decrease. The interaction mechanisms between model compartments are schematically described in Figure 1 Notably, we chose to individually model each mechanism in order to better characterize it.

We assumed that a given dose of activated CAR T cells is introduced into the system as an adoptive therapy. The effector CAR $\mathrm{T}$ cells have antitumor activity owed to a specific chimeric antigen receptor (CAR) against an antigen expressed by the tumor 33, 38. The overall net population balance of the effector CAR T cells depends on their spontaneous death and proliferation, inhibition by the tumor cells, and differentiation to a memory CAR T cell phenotype [34, 37. This means that a portion of CAR $\mathrm{T}$ cells persists as memory $\mathrm{T}$ cells that keep antitumor antigen specificity and patient characteristic [21, 31. They have a lower activation threshold, which eases the secondary response to a future tumor recurrence 35 . They also have a long half-life, providing long-term protection. In the absence of immunosurveillance, the tumor grows exponentially 
bioRxiv preprint doi: https://doi.org/10.1101/779793; this version posted April 18, 2020. The copyright holder for this preprint (which was

but we also assumed that the growth rate decreases linearly with the population size, approaching zero as the tumor population approaches the maximal tumor burden (carrying capacity) [8, 28. Upon contact, effector CAR T cells kill tumor cells. As the rate of CAR T cell killing capacity could not be directly measured in vivo, we assumed the constant rate as a range of tumor cell killing by effector CAR T cells over time [14, 17, 3]. .

The change of each cell population in time depends on the balance among all factors contributing to its increase and decrease. Specifically, the dynamics of the effector CAR T cells is described by:

$\frac{d C_{T}}{d t}=\phi C_{T}-\rho C_{T}-\mu_{T} C_{T}+\theta T C_{M}-\alpha T C_{T}$.

The right-hand side term specifies that effector CAR T cells undergo expansion due to proliferation at a rate of $\phi$, and have half-life $1 / \mu_{T}$. According to the linear progression model described in [5, 12, they differentiate at a rate of $\rho$ into long-term CAR T memory cells, which are assumed to provide long-lasting protection to the specific tumor/antigen. At any future time in which memory CAR $\mathrm{T}$ cells come into contact with the same tumor cells, they can rapidly be converted into effectors CAR $\mathrm{T}$ cells, readily activated to prevent tumor progression. This feature is modeled by the term $\theta T C_{M}$ represents. Finally, the term $\alpha T C_{T}$ models tumor-modulated CAR inhibition that can encompasses a variety of immunosuppressive mechanisms.

The dynamics of the immunological memory, a key dynamic of the adaptive immune system [5, 36] is modeled by

$\frac{d C_{M}}{d t}=\epsilon \rho C_{T}-\theta T C_{M}-\mu_{M} C_{M}$.

Since we are dealing with immunodeficient mice, memory $\mathrm{T}$ cells are formed exclusively from the differentiation of CAR $\mathrm{T}$ cells at a rate of $\epsilon \rho$. When in future contact with the same antigen-bearing cancer cells, they immediately return to the effector CAR T cell phenotype at a per capita rate proportional to the tumor burden. In general, memory $\mathrm{T}$ cells have longevity, and therefore have a much lower mortality rate than the effector T cells 36, i.e., $\mu_{M}<<\mu_{T}$.

The response of tumor cells to the CAR T immunotherapy is modeled by

$\frac{d T}{d t}=r T(1-b T)-\gamma T C_{T}$

The first term in the right-hand side of (3) models the density-dependent growth of cancer cells due to the limitation of available resources in the tumor microenvironment. Tumor growth is described using a logistic function in which $r$ is the maximum growth rate and
$1 / b$ is the carrying capacity, i.e., the maximum cell density that the available resources are capable of sustaining. CAR T cell immunotherapy acts by inhibiting tumor growth by cytotoxic action, causing a per-capita mortality rate of $\gamma C_{T}$ that depends on the number of effector T cells.

The meaning of the parameters (strictly positive real numbers) is given in Table 1 . Notice that model (1)-(3) has ten parameters. Their estimation ultimately defines the desired immunotherapy scenario. Of note, the linear same order terms in equation (1) can be combined to reduce the number of parameters. This procedure is particularly useful for cases where experimental data is scarce, for which a single rate may be alternatively used to reproduce the balanced effect of proliferation, death, and differentiation of effector CAR T cells. Details about parameter inference are presented in the Supplementary Material. The next section shows that the overall approach provides a framework for investigating the roles of CAR T cell dose, immune memory, immunosuppressive tumor microenvironment and individual uncertainties on the therapy response.

\section{Results: In silico experiments}

Mathematical equations (1)-(3) were solved numerically using the explicit fourth-order Runge-Kutta method 9. Simulations represent CAR T cell immunotherapy performed in immunodeficient mice previously injected with tumor cells. This amounts to set the initial condition for the tumor population, $T(0)$, as the injected tumor cells, and $C_{T}(0)=C_{M}(0)=0$ cells. At the time the immunotherapy is given, when tumor burden has already undergone significant growth, $C_{T}$ receives the CAR T cells dose. Cell populations are followed up to investigate tumor response and immunological memory formation.

CAR T 123 therapy eliminates HDLM-2 tumor, providing long-term protection; the immunotherapy with CAR T 19 on Raji tumor slows down its growth

We first simulate the scenario presented in 30. that consists of CAR T 123 therapy against HDLM-2 cells. Ruella et al. 30] reported that $2 \times 10^{6}$ cells of Hodgkin lymphoma (HDLM-2) were injected into NSG mice. After 42 days, mice received $C_{T}=2 \times 10^{6}$ cells of CAR T 123 immunotherapy, which eliminated tumors very rapidly. Model parameters are displayed in Table S6 of the Supplementary Material. Simulation begins with $T(0)=2 \times 10^{6}$ HDLM-2 cells, and tumor progresses in time until it reaches about $2 \times 10^{7}$ cells in $t=42$ days 
bioRxiv preprint doi: https://doi.org/10.1101/779793; this version posted April 18, 2020. The copyright holder for this preprint (which was

(Figure 2(a)). At this time, immunotherapy with CAR $\mathrm{T} 123$ is performed, so that $C_{T}=2 \times 10^{6}$ cells at $t=42$ days. Immunotherapy rapidly eliminates the population of tumor cells in a few days, retrieving the experimental remission results presented in 30. Our simulation also provides the dynamics of effector and memory $\mathrm{T}$ cells. Figure 2(a) shows that, as the population of $C_{T}$ cells decrease, phenotypic differentiation occurs giving rise to memory $\mathrm{T}$ cells $C_{M}$. Our simulation shows that effector CAR T cell populations remain undetectable until $t=250$ days, which agrees on results presented in 30 around ten months after the injected dose. Moreover, our model indicates the presence of long-term memory CAR T cells, which slightly decline in time due to small mortality rate of $\mu_{M}$.

In an additional experiment, Ruella et al. 30] demonstrated the formation of the immune memory by challenging previously treated mice with $1 \times 10^{6}$ HDLM2 cells at $t=250$ days. The tumor remained undetectable, being rejected due to the re-expansion of the effector CAR T cells. To interrogate the model on this behavior, we continue the previous simulation by introducing $1 \times 10^{6}$ tumor cells at $t=250$ days. Figure 2 (a) shows how the model answers such a challenge. The presence of tumor cells drives the conversion of $C_{M}$ into $C_{T}$ cells which are rapidly able to eliminate the new tumor. Afterward, $C_{T}$ undergoes rapid decay while part of the memory $\mathrm{T}$ cells population is recovered. Tumor clearance remains until the end of simulation on day 500. As explained in 30, tumor rejection occurs due to the re-expansion of previously undetectable effector CAR T cells.

We next fit the model to the scenario described in [29, regarding Raji tumor and immunotherapy with CAR T 19 cells. Raji tumors are much more aggressive than HDLM tumors and express the CD19 antigen. Ninomiya et al. 29] reported that $3 \times 10^{6}$ Raji tumor cells were injected in SCID/Beige mice and therapy with $1 \times 10^{7}$ cells of CAR T 19 cells was given in day 7 , which did not eliminate the tumor but could control its growth. This scenario is simulated with the estimated parameters displayed in Table S6 of the Supplementary Material. Beginning with $T(0)=3 \times 10^{6}$ cells, the tumor reaches almost $1 \times 10^{8}$ cells at day 7 when $C_{T}=1 \times 10^{7}$ cells of CAR T 19 cells is introduced. Retrivieng the results presented in [29], the immunotherapy is able to reduce the tumor growth rate but not eliminate it, and tumor cell population reaches $6 \times 10^{8}$ cells at day 14 , as shown in Figure 2(b). The effector $\mathrm{T}$ cells undergo an expansion of about $30 \%$ on day 9 , from which they decrease to extinction, representing the $\mathrm{T}$ cell time course reported in [29]. The immunotherapy dose is not enough to lead to the formation of memory CAR T cells.
Insights on CAR T 123 dosing strategy into the elimination of HDLM-2 tumors

The model was used now to investigate how the relationship between tumor burden and CAR T cell dose and injection protocol impact therapy outcomes. To first assess how the dose interferes with the response to the CAR T 123 immunotherapy, we perform three different simulations with therapeutic doses of $1 \times 10^{6}$, $5 \times 10^{5}$ and $2 \times 10^{5}$ cells. We use the same scenario described in Figure 2 (a) and same model parameters, keeping the initial tumor burden equal to $T(0)=2 \times 10^{6}$ cells. The resulting dynamics are shown in Figure 3(a)(c). A CAR T dose of $1 \times 10^{6}$ cells can perform tumor elimination, although the level of memory $\mathrm{T}$ cells at $t=200$ days is smaller than that in the case presented in Figure 2(a). Higher CAR T cell dose generates greater immunological memory $\mathrm{T}$ cell pool. On the other hand, reducing the dose of CAR $\mathrm{T}$ cell to $5 \times 10^{5}$ cells, the tumor is not completely eliminated. It undergoes an intense decrease but resumes growth at day 150, eventually reaching a state in which it does not grow or shrink significantly, wherein the tumor is reduced to a very small (but not zero) value as depicted in Figure 3(b). In this equilibrium state, both $C_{T}$ and $C_{M}$ $\mathrm{s}$ are non-zero, and therefore there is the coexistence of the three cell populations. This is a typical configuration of tumor dormancy. Finally, reducing, even more, the CAR T dose to $2 \times 10^{5}$ cells, the tumor escapes from the immunotherapy. Figure 3(c) shows that the tumor is initially reduced by therapy (not visualized because of the scale) but resumes growth and reaches the carrying capacity at around day 300 . There is a complete and rapid extinction of the CAR $\mathrm{T}$ population and no formation of memory CAR $\mathrm{T}$ cells.

Although not shown, it is worth remarking that those three tumor responses of elimination, equilibrium, and escape can be reached by fixing the CAR $\mathrm{T}$ dose and increasing the tumor burden.

The next experiment explores the alternative possibility of CAR T cell dose fractionation. We select the same scenario described in Figure 2(a) with 1-time infusion of $2 \times 10^{6}$ cells, which promotes tumor elimination. Firstly, simulations are performed dividing the total dose into four equal fractions, infused at every seven or fourteen days. Figures 3 (d) and 3(e) show that the dosing split does not interfere on the tumor elimination, which occurs in few days. Of note, a single dose of $5 \times 10^{5}$ CAR T cells is not able to eliminate the tumor burden, as shown in Figure 3(b). While in a single infusion case tumor decreases but resumes growth until reaching equilibrium, the used fractionated infusions prevent tumor regrowth. As well as in Figure 2(a), immunolog- 
bioRxiv preprint doi: https://doi.org/10.1101/779793; this version posted April 18, 2020. The copyright holder for this preprint (which was

ical memory is formed, and the peak of memory cells is similar to that of single total dose infusion, although a certain delay is observed due to dose fractionation. Such delay ultimately yields a greater formation of immunological memory on day 200. Specifically, the number of memory CAR T cells at that time is $10 \%$ and $17 \%$ for 7 and 14 days rest time between doses, respectively. Although this feature could be seen as an advantage towards fractionated infusions, long rest periods between doses cannot be used because CAR T cells do not survive in culture medium for such long times. Alternatively, a simulation is performed for a more realistic fractionated immunotherapy, as investigated in [10. In that work, patients with relapsed or refractory CD19+ acute lymphoblastic leukemia were treated with three fractionated infusions over 3 days with increasing doses (10\%:d1, 30\%:d2, and 60\%:d3). It was shown that such treatment protocol does not compromise effectiveness while reducing toxicity effects. Figure 3 (f) shows the in silico predictions using this protocol. Like in a 1-time infusion protocol shown in Figure 2(a), tumor is rapidly eliminated, effector $\mathrm{T}$ cells vanish in 100 days while immunologic memory amounts for $1.5 \times 10^{6}$ cells at day 200.

How do parameter uncertainties impact the elimination of HDLM-2 tumors?

We now use in silico experiments to investigate how parameter uncertainties impact the CAR T $123 \mathrm{im}$ munotherapy outcomes. We selected the scenario depicted in Figure 2(a): HDLM-2 tumor burden of $T(0)=$ $2 \times 10^{6}$ cells, CAR T 123 dose of $2 \times 10^{6}$ cells introduced at day 42 , and the set of parameters depicted in Table S6 of the Supplementary Material. Model simulation under these conditions leads to tumor elimination. We then considered that parameter values are uncertain. Recalling that parameter values were estimated for the scenario defined in [30, uncertainty can be regarded as associated with individual characteristics of each mouse, tumor cell lineage, and CAR T cell donor. Since we did not have any evidence of such uncertainties, we assumed that each parameter is a random variable with uniform distribution in the range limited by $20 \%$ of the reference values indicated in Table S6. We built 10,000 sets of parameters from randomly selecting samples of the parameter distributions. We then simulated the model for each set of parameters in order to assess if small random variations in the parameter values can impact tumor elimination. We verified that they indeed impact the therapy outcome, and we registered the elimination, equilibrium or escape responses. The simulations indicated that the uncertainties in the parameters can drastically reduce the chance of therapy success: of the 10,000 cases considered, the therapy was successful in only $5 \%$ of them (507 cases). The equilibrium and escape responses occurred in $18 \%$ and $77 \%$ of the total 10,000 cases, respectively. Overall, the performed simulations indicate that tumor response is very sensitive to parameter uncertainties, and tumor escape is most likely to occur. To assess how each parameter value impacts these responses, we then built the three heatmaps shown in Figure 4 , that displays the frequency of occurrence of each outcome (elimination, equilibrium, and escape) with respect to the parameter values. The inspection of these plots indicates that the elimination heatmap is more heterogeneous while the escape one is more homogeneous. The latter indicates that all parameters contribute more uniformly to this outcome, meaning that none of the parameters plays a significant differential role in tumor escape. On the other hand, the more heterogeneous occurrence frequencies in the elimination heatmap allow identification that the tumor proliferation $(r)$, CAR T cell inhibition $(\alpha)$, and CAR T cell proliferation $(\phi)$ and death $\left(\mu_{T}\right)$ are the most influential for tumor elimination.

The effect of inhibitors of immunosuppressive tumor microenvironments

Our model includes the term $\alpha T C_{T}$ in Equation (1) to describe tumor-modulated immunosuppressive mechanisms. Higher $\alpha$ value implies in a stronger immunosuppressive mechanism. To check how this term allows investigating the blocking action of these mechanisms and, at the same time, how the model deals with different tumors and CAR T cells, we selected data from 29 that presents the action of CAR T 19 cell immunotherapy against CD19 + lymphoma that expresses the IDO enzyme in mice. We then considered the Raji-IDO tumor when treated with CAR T 19 cells alone or combined with 1-MT, an IDO inhibitor. We estimated $\alpha$ for these scenarios, keeping all other parameters fixed with values shown in Table S6 for the Raji tumor (control). It should be noted that, according to Figure $2 \mathrm{C}$ of 29], Raji-control and Raji-IDO tumor sizes on the day of immunotherapy administration are indistinguishable so that the same tumor proliferation rate was used. Figure 5 shows system responses for the Raji-IDO + CAR T 19 and Raji-IDO + CAR T $19+$ 1-MT scenarios, together with the most likely values (MLEs) obtained for $\alpha$. The smaller $\alpha$ value obtained when 1-MT was used allowed a greater expansion of CAR T cells after infusion which in turn provided a stronger control on the tumor growth than that promoted by the CAR T cells without 1-MT. Of note, in both cases the CAR T 19 
bioRxiv preprint doi: https://doi.org/10.1101/779793; this version posted April 18, 2020. The copyright holder for this preprint (which was

dose was not able to eliminate the tumor, which eventually escapes, and there is no formation of memory CAR T cells. We can also notice the similarity of $\alpha$ values for the Raji-control + CAR T 19 and Raji-IDO + CAR T $19+1-\mathrm{MT}$, reflecting the ability of the 1-MT to block the immunosuppressive effect of the IDO. Thus, the model could capture the effect of the IDO inhibitor through the $\alpha$ parameter that can actually modulate the immunosuppression mechanism used by Raji-IDO tumors. These simulations show the ability of $\alpha$ in modulating immunosuppressive mechanisms displaying the potential use of our mathematical model as an adjuvant in silico platform to test immune checkpoint inhibitors.

\section{Discussion}

CAR T cell therapies are spreading across hematological cancers and are already a product of big pharma companies 25. On the road, there are new CAR designs, including new antigen targets, different CAR affinity [15], and expansion protocols [7].

We developed a three-compartment mathematical model to describe tumor response to CAR T cell immunotherapy in immunodeficient mouse models (NSG and SCID/beige) based on two published articles from literature. In a general CAR T cell therapy model, independently of the recognized antigen, we modeled different receptors as CART 19BBz and CART 123, and also different tumor targets as HDLM-2 and Raji. The HDLM-2 tumor model was used as a low proliferation, less aggressive tumor model where CAR T cell therapy is effective on tumor elimination. On the other hand, the Raji model was chosen from its high proliferation and escape from CAR T cell therapy. On the Raji model we also included explicitly immune checkpoint inhibitor molecule as IDO in order to estimate this component on CAR $\mathrm{T} /$ tumor cell interaction. Therefore, the model could adapt itself to different treatment and tumor scenarios. The adopted structure of our mathematical model allows identifying each individual mechanism in a more transparent way. Donor/tumormicroenvironment specificities were considered as uncertainties in the parameters of the model, which were shown to greatly impact the therapy outcome. The model was able to represent tumor elimination after immunotherapy with CAR T 123 cells even in case of a new tumor challenge due to memory $\mathrm{T}$ cells' long-term protection for HDLM-2 target. The change of CAR T cells from effector to memory cells and their long-term persistence as CAR T memory cells were also demonstrated by our previous work with RS4;11 B-ALL model using 19BBz CAR T 4 . For the CAR T 19 therapy and Raji target scenarios, the model represented well the tumor dynamics with or without IDO inhibitor. We performed a few in silico studies to highlight how the model can be used as an adjuvant platform to contribute to a better understanding of the underlying processes. We found that the determination of the dose of CAR $\mathrm{T}$ for a given tumor burden is a critical factor for the success of the immunotherapy. A previous model already considered CAR $\mathrm{T}$ cell proliferation in response to antigen burden, but memory CAR $\mathrm{T}$ was not considered, neither the effect of tumor inhibition of CAR T cells [37. Another interesting mathematical model was made upon tisagenlecleucel-treated patient data [34]. This model was adapted from a previous empirical model of an immune response to bacterial/viral infections. They captured $\mathrm{T}$ cell expansion, contraction, and persistence like our model does, including CAR T memory population. Their model was calibrated on patients' data, and different from ours, no difference in dose-response was detected. They attributed this result to CAR T cell proliferation capacity in vivo. We partially agree, but there is a possibility that obtained data from humans do not present very different CAR $\mathrm{T}$ cell dose (especially including only tisagenlecleucel clinical trials). Considering mouse model data, where CAR $\mathrm{T}$ cell dose varies by thousands, we do observe a dose effect, especially on aggressive, high proliferative tumors as NALM-6 cell line [7, 4].

Another advantage of our mathematical model is the calculation of therapy effectiveness. Overall therapy effectiveness may depend on intrinsic individual specificities, regarded here as small variations in the model parameters' values. In the studied case, such parameter uncertainties drastically reduced the chance of tumor elimination to less than $10 \%$. Additional in silico experiments can be conducted to identify, for example, the smallest dose to increase success chance in view of a setting with possible uncertainties. We have also shown that fractionation of dose appears to be as effective as a single dose, and the rest periods between infusions might favor long-term immunological memory. These results corroborate with previous clinical trials using fractioned CAR T cell dose with similar effectiveness to single-dose and persistence of CAR $\mathrm{T}$ cells on the blood 20 months after therapy [25].

We identified that uncertainties associated with the tumor proliferation and ability to inhibit the CAR T cells, and CAR T cell proliferation and death are the most significant to therapy success in eliminating the tumors. This opens room for investigating other chimeric antigen T-cell receptors with different target antigen affinities and the blockade of immune checkpoints to boost efficacy and safety. In our model, we did not con- 
bioRxiv preprint doi: https://doi.org/10.1101/779793; this version posted April 18, 2020. The copyright holder for this preprint (which was

sider CAR $\mathrm{T}$ affinity for each antigen as an explicit parameter, considering it as a result of tumor lysis by CAR T cells. Another aspect that we did not take on consideration is the toxicity effect of CAR T cell therapy (cytokine release syndrome - CRS) because our model is based on an immunodeficient mouse model that lacks this effect. For human data, Hanson et al. [18] made a mathematical model to CAR $\mathrm{T}$ cell therapy for B-ALL emphasizing cytokines and CRS, but also considering CAR T effector and memory cells.

Overall, the developed mathematical model may help to shed light on the structure of the treatment protocol. To this end, the model must be calibrated by using one in vivo experimental data describing the tumor growth without and with treatment, and in vitro lysing data. Once calibrated, the model allows exploring alternative ways of scheduling and infusion dose in view of the current setting specificities, including parameter uncertainties, so as to elicit the one with a higher chance of success. The model provides an in silico tool for assessing different issues associated with the therapy such as how CAR $\mathrm{T}$ cell dosing can be adjusted according to tumor burden, CAR T cell infusion protocols, immunosuppressive mechanisms, among others, without further in vivo experiments.

Conflict of interest The authors declare that the research was conducted in the absence of any commercial or financial relationships that could be construed as a potential conflict of interest.

Acknowledgments The authors thank the support granted by FAPERJ and CAPES.

\section{References}

1. Ashley B. Allen, Zulma Gazit, Susan Su, Hazel Y. Stevens, and Robert E. Guldberg. In vivo bioluminescent tracking of mesenchymal stem cells within large hydrogel constructs. Tissue Engineering: Part C, 20(10):806 - 816, 2012.

2. Rustom Antia, Vitaly V. Ganusov, and Rafi Ahmed. The role of models in understanding CD8+ T-cell memory. Nature Reviews Immunology, 5:101 $-111,2005$.

3. Mohamed-Reda Benmebarek, Clara Helke Karches, Bruno Loureiro Cadilha, Stefanie Lesch, Stefan Endres, and Sebastian Kobold. Killing mechanisms of chimeric antigen receptor (CAR) T cells. International Journal of Molecular Sciences, 20:1283, 2019.

4. Leonardo Chicaybam, Luiza Abdo, Mayra Carneiro, Bárbara Peixoto, Mariana Viegas, Priscila de Sousa, Márcia C. Fornazin, Maria C.
Spago, Angelo Brunelli Albertoni Laranjeira, Pedro O. de Campos-Lima, Alexandre Nowill, Luciana Rodrigues Carvalho Barros, and Martín Hernán Bonamino. CAR T cells generated using sleeping beauty transposon vectors and expanded with an EBV-transformed lymphoblastoid cell line display antitumor activity in vitro and in vivo. Human Gene Therapy, 30(4):511 - 522, 2019. PMID: 30793967.

5. Fabien Crauste, Julien Mafille, Lilia Boucinha, Sophia Djebali, Olivier Gandrillon, Jacqueline Marvel, and Christophe Arpin. Identification of nascent memory CD8 T cells and modeling of their ontogeny. Cell Systems, 4:306 - 317, 2017.

6. Katherine D. Cummins and Saar Gill. AntiCD123 chimeric antigen receptor T-cells (CART): an evolving treatment strategy for hematological malignancies, and a potential ace-in-the-hole against antigen-negative relapse. Leukemia 83 Lymphoma, 59(7):1539 - 1553, 2018. PMID: 28901790.

7. Luiza de Macedo Abdo, Luciana Rodrigues Carvalho Barros, Mariana Saldanha Viegas, Luisa Vieira Codeço Marques, Priscila de Sousa Ferreira, Leonardo Chicaybam, and Martín Hernán Bonamino. Development of car-t cell therapy for b-all using a point-of-care approach. bioRX iv The Preprint Server for Biology, 2019.

8. Heiko Enderling and Mark A.J. Chaplain. Mathematical modeling of tumor growth and treatment. Current Pharmaceutical Design, 20(30):4934 - 4940, 2014.

9. J. Douglas Faires and Richard L. Burden. Numerical Methods. Cengage Learning, Belmont, CA, USA, 2003.

10. Noelle V. Frey, Pamela A Shaw, Elizabeth O. Hexner, Saar Gill, Katherine Marcucci, Selina M. Luger, James K. Mangan, Stephan A. Grupp, Shannon L. Maude, Solveig Ericson, Bruce Levine, Simon F. Lacey, Jan J. Melenhorst, Carl H. June, and David L. Porter. Optimizing chimeric antigen receptor (CAR) T cell therapy for adult patients with relapsed or refractory $(\mathrm{r} / \mathrm{r})$ acute lymphoblastic leukemia (ALL). Journal of Clinical Oncology, 34(15_suppl):7002, 2016.

11. G3Data, 1991. G3Data Graph Analyzer, Available at https://github.com/pn2200/g3data, Acessed in 13-07-2018.

12. Vitaly V. Ganusov. Discriminating between different pathways of memory $\mathrm{CD} 8^{+} \mathrm{T}$ cell differentiation. The Journal of Immunology, 179:5006 - 5013, 2007.

13. Julie Gertner-Dardenne. Standard 4-hours chromium-51 $\left({ }^{51} \mathrm{cr}\right)$ release assay. Bio-protocol, 
bioRxiv preprint doi: https://doi.org/10.1101/779793; this version posted April 18, 2020. The copyright holder for this preprint (which was

not certified by peer review) is the author/funder, who has granted bioRxiv a license to display the preprint in perpetuity. It is made available under aCC-BY-NC-ND 4.0 International license.

2(23):e201, 2012.

14. Mark B. Geyer and Renier J. Brentjens. Review: Current clinical applications of chimeric antigen receptor (CAR) modified T cells. Cytotherapy, 18(11):1393 - 1409, 2016.

15. S. Ghorashian, A. M. Kramer, S. Onuoha, Wright G., et al. Enhanced CAR T cell expansion and prolonged persistence in pediatric patients with ALL treated with a low-affinity CD19 CAR. Nature Medicine, 25(9):1408 - 1414, 2019.

16. Sonia Guedan, Marco Ruella, and Carl H. June. Emerging cellular therapies for cancer. Annual Review of Immunology, 37(1):145 - 171, 2019. PMID: 30526160.

17. Dora Hammerl, Dietmar Rieder, John W.M. Martens, Zlatko Trajanoski, and Reno Debets. Adoptive T cell therapy: New avenues leading to safe targets and powerful allies. Trends in Immunology, 39(11):921 - 936, 2018.

18. Shalla Hanson, David Robert Grimes, Jake P. Taylor-King, Benedikt Bauer, Pravnam I. Warman, Ziv Frankenstein, Artem Kaznatcheev, Michael J. Bonassar, Vincent L. Cannataro, Zeinab Y. Motawe, Ernesto A. B. F. Lima, Sungjune Kim, Marco L. Davila, and Arturo Araujo. Toxicity management in CAR T cell therapy for B-ALL: Mathematical modelling as a new avenue for improvement. bioRX iv The Preprint Server for Biology, 2016.

19. E. Jacoby. Relapse and resistance to CAR-T cells and blinatumomab in hematologic malignancies. Clinical Hematology International, 1(2):79 - 84, 2019.

20. Joanneke E. Jansen, Eamonn A. Gaffney, Jonathan Wagg, and Mark C. Coles. Combining mathematical models with experimentation to drive novel mechanistic insights into macrophage function. Frontiers in Immunology, 10:1283, 2019.

21. Michael Kalos, Bruce L. Levine, David L. Porter, Sharyn Katz, Stephan A. Grupp, Adam Bagg, and Carl H. June. T cells with chimeric antigen receptors have potent antitumor effects and can establish memory in patients with advanced leukemia. Science Translational Medicine, 3(95):95ra73, 2011.

22. Anna Konstorum, Anthony T. Vella, Adam J. Adler, and Reinhard C. Laubenbacher. Addressing current challenges in cancer immunotherapy with mathematical and computational modelling. Journal of the Royal Society Interface, 14(131):20170150, 2017.

23. Natalie Kronik, Yuri Kogan, Vladimir Vainstein, and Zvia Agur. Improving alloreactive CTL immunotherapy for malignant gliomas using a simu- lation model of their interactive dynamics. Cancer Immunology, Immunotherapy, 57(3):425 - 439, 2008.

24. Kalet Leon, Karina Garcia-Martinez, and Tania Carmenate. Mathematical models of the impact of IL2 modulation therapies on $\mathrm{T}$ cell dynamics. Frontiers in Immunology, 4:439, 2013.

25. Shannon L. Maude, Theodore W. Laetsch, Jochen Buechner, Susana Rives, Michael Boyer, Henrique Bittencourt, Peter Bader, Michael R. Verneris, Heather E. Stefanski, Gary D. Myers, Muna Qayed, Barbara De Moerloose, Hidefumi Hiramatsu, Krysta Schlis, Kara L. Davis, Paul L. Martin, Eneida R. Nemecek, Gregory A. Yanik, Christina Peters, Andre Baruchel, Nicolas Boissel, Francoise Mechinaud, Adriana Balduzzi, Joerg Krueger, Carl H. June, Bruce L. Levine, Patricia Wood, Tetiana Taran, Mimi Leung, Karen T. Mueller, Yiyun Zhang, Kapildeb Sen, David Lebwohl, Michael A. Pulsipher, and Stephan A. Grupp. Tisagenlecleucel in children and young adults with b-cell lymphoblastic leukemia. New England Journal of Medicine, 378(5):439 - 448, 2018. PMID: 29385370.

26. Shannon L Maude, David T Teachey, David L Porter, and Stephan A Grupp. CD19-targeted chimeric antigen receptor $\mathrm{T}$ cell therapy for acute lymphoblastic leukemia. Blood, pages 4017 - 4023, 2015.

27. Shannon L. Maude, David T. Teachey, Susan R. Rheingold, Pamela A Shaw, Richard Aplenc, David Maxwell Barrett, Christine S Barker, Colleen Callahan, Noelle V. Frey, Farzana Nazimuddin, Simon F. Lacey, Zhaohui Zheng, Bruce Levine, Jan Joseph Melenhorst, Laura Motley, David L. Porter, Carl H. June, and Stephan A. Grupp. Sustained remissions with CD19-specific chimeric antigen receptor (CAR)-modified $\mathrm{T}$ cells in children with relapsed/refractory ALL. Journal of Clinical Oncology, 34(15_suppl):3011, 2016.

28. Hope Murphy, Hana Jaafari, and Hana M. Dobrovolny. Differences in predictions of ODE models of tumor growth: a cautionary example. BMC Cancer, 16(1):163, 2016.

29. Soranobu Ninomiya, Neeharika Narala, Leslie Huye, Shigeki Yagyu, Barbara Savoldo, Gianpietro Dotti, Helen E. Heslop, Malcolm K. Brenner, Cliona M. Rooney, and Carlos A. Ramos. Tumor indoleamine 2, 3-dioxygenase (IDO) inhibits CD19CAR T cells and is downregulated by lymphodepleting drugs. Blood, pages 3905 - 3916, 2015.

30. Marco Ruella, Michael Klichinsky, Saad S. Kenderian, Olga Shestova, Amy Ziober, Daniel O. Kraft, 
bioRxiv preprint doi: https://doi.org/10.1101/779793; this version posted April 18, 2020. The copyright holder for this preprint (which was not certified by peer review) is the author/funder, who has granted bioRxiv a license to display the preprint in perpetuity. It is made available under aCC-BY-NC-ND 4.0 International license.

Michael Feldman, Mariusz A. Wasik, Carl H. June, and Saar Gill. Overcoming the immunosuppressive tumor microenvironment of Hodgkin Lymphoma using chimeric antigen receptor T cells. Cancer Discovery, 10:1154 - 1167, 2017.

31. Marianna Sabatino, Jinhui Hu, Michele Sommariva, Sanjivan Gautam, Vicki Fellowes, James D. Hocker, Sean Dougherty, Haiying Qin, Christopher A. Klebanoff, Terry J. Fry, Ronald E. Gress, James N. Kochenderfer, David F. Stroncek, Yun Ji, and Luca Gattinoni. Generation of clinical-grade cd19-specific car-modified cd8+ memory stem cells for the treatment of human b-cell malignancies. Blood, 128(4):519 - 528, 2016.

32. Michel Sadelain, Isabelle Rivière, and Stanley Riddell. Therapeutic $\mathrm{T}$ cell engineering. Nature, 545:423 - 431, 2017.

33. Daniel Sommermeyer, Michael Hudecek, Paula L Kosasih, Tea Gogishvili, David G Maloney, Cameron J Turtle, and Stanley R Riddell. Chimeric antigen receptor-modified $\mathrm{T}$ cells derived from defined CD8+ and CD4+ subsets confer superior antitumor reactivity in vivo. Leukemia, 30(2):492, 2016.

34. Andrew M. Stein, Stephan A. Grupp, John E. Levine, Theodore W. Laetsch, Michael A. Pulsipher, Michael W. Boyer, Keith J. August, Bruce L. Levine, Lori Tomassian, Sweta Shah, Mimi Leung, Pai-Hsi Huang, Rakesh Awasthi, Karen Thudium Mueller, Patricia A. Wood, and Carl H. June. Tisagenlecleucel model-based cellular kinetic analysis of chimeric antigen receptor-T cells. CPT: Pharmacometrics 6 Systems Pharmacology, 8(5):285 - 295, 2019.

35. Corinne Tanchot, François A Lemonnier, Beatrice Pérarnau, Antonio A Freitas, and Benedita Rocha. Differential requirements for survival and proliferation of CD8 naive or memory T cells. Science, 276(5321):2057 - 2062, 1997.

36. Andre Tanel, Simone G Fonseca, Bader YassineDiab annd Rebeka Bordi, Joumana Zeidan, Yu Shi, Clarisse Benne, and Rafick-Pierre Sékaly. Cellular and molecular mechanisms of memory T-cell survival. Expert Review of Vaccines, 8(3):299 - 312, 2009.

37. Amir A. Toor, Alden Chesney, Jamal Zweit, Jason Reed, and Shahrukh K. Hashmi. A dynamical systems perspective on chimeric antigen receptor T-cell dosing. Bone Marrow Transplantation, 54(3):485 - 489, 2019.

38. Cameron J Turtle, Laïla-Aïcha Hanafi, Carolina Berger, Theodore A Gooley, Sindhu Cherian, Michael Hudecek, Daniel Sommermeyer, Katherine
Melville, Barbara Pender, Tanya M Budiarto, et al. CD19 CAR-T cells of defined CD4+: CD8+ composition in adult B cell ALL patients. The Journal of Clinical Investigation, 126(6):2123 - 2138, 2016.

39. Veronika I. Zarnitsyna, Andreas Handel, Sean R. McMaster, Sarah L. Hayward, Jacob E. Kohlmeier, and Rustom Antia. Mathematical model reveals the role of memory CD8 $\mathrm{T}$ cell populations in recall responses to influenza. Frontiers in Immunology, $7: 165,2016$.

\section{Figure captions}


bioRxiv preprint doi: https://doi.org/10.1101/779793; this version posted April 18, 2020. The copyright holder for this preprint (which was not certified by peer review) is the author/funder, who has granted bioRxiv a license to display the preprint in perpetuity. It is made available under aCC-BY-NC-ND 4.0 International license.

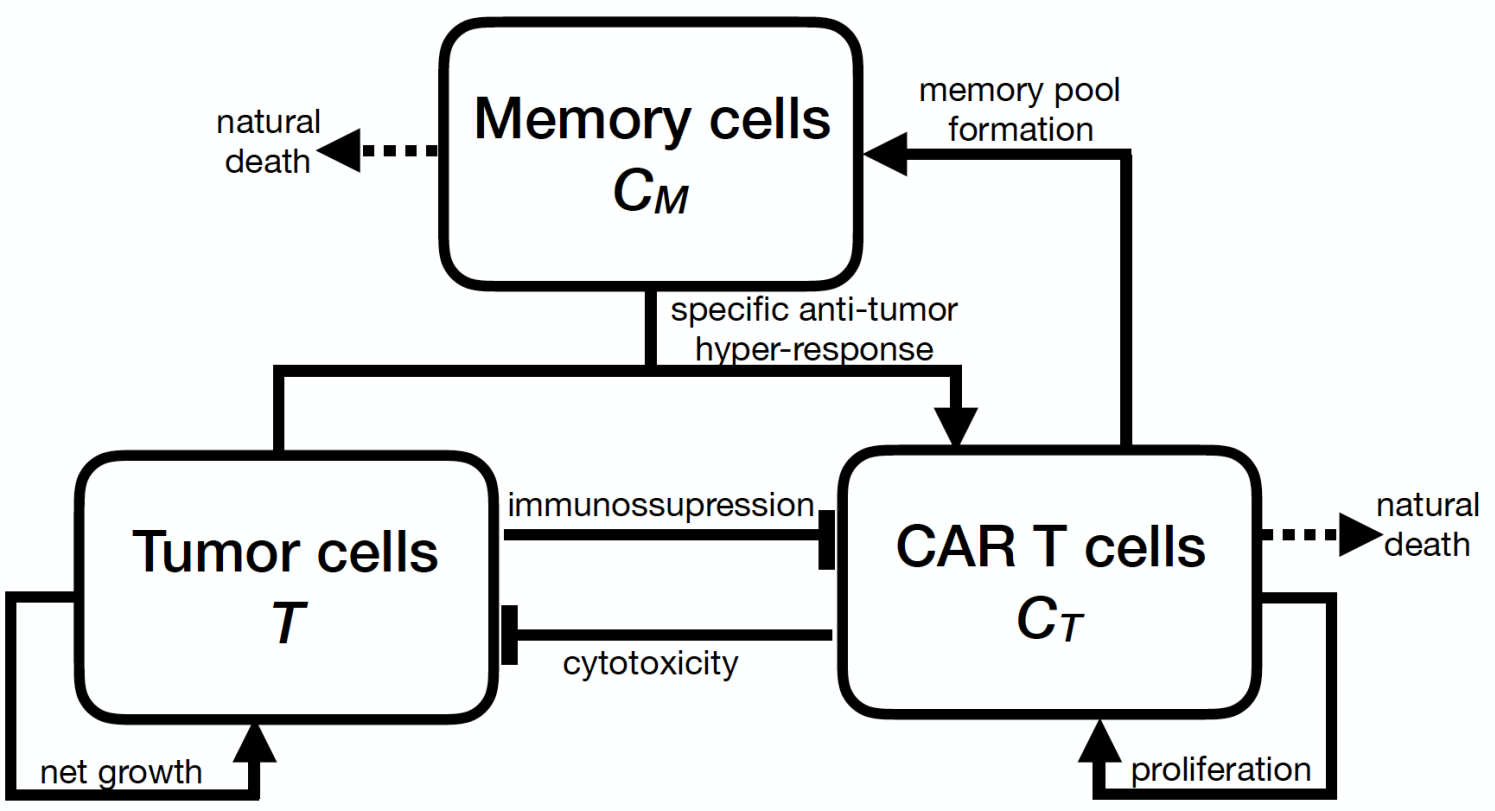

Fig. 1 Schematic description of the model structure. CAR T cells proliferate, have a cytotoxic effect on tumor cells, differentiate into memory cells, and die naturally or due to immunosuppressive mechanisms. The long-lived memory $\mathrm{T}$ cells also die naturally and are readily responsive to the tumor associated antigen so that, when they interact with tumor cells, they differentiate into effector $\mathrm{T}$ cells, producing a rapid response of the immune system.

Table 1 Summary of the model parameters.

\begin{tabular}{cll}
\hline Parameter & Meaning & Unit \\
\hline$\phi$ & $C_{T}$ proliferation rate & day $y^{-1}$ \\
$\rho$ & Differentiation rate of $C_{T}$ into $C_{M}$ & $d a y^{-1}$ \\
$\mu_{T}$ & $C_{T}$ death rate & $d a y^{-1}$ \\
$\theta$ & Conversion coefficient of $C_{M}$ into $C_{T}$ due to interaction with $T$ & $(\text { cell } \cdot \text { day })^{-1}$ \\
$\alpha$ & $C_{T}$ inhibition coefficient due to interation with $T$ & $(\text { cell } \cdot \text { day })^{-1}$ \\
$\epsilon$ & Numerical response of the conversion of $C_{T}$ into $C_{M}$ & - \\
$\mu_{M}$ & $C_{M}$ death rate & day $y^{-1}$ \\
$r$ & Maximum growth rate of $T$ & day $y^{-1}$ \\
$b$ & Inverse of the tumor carrying capacity & cell $l^{-1}$ \\
$\gamma$ & Death coefficient induced by $C_{T}$ & $\left({\text { cell } \cdot d a y)^{-1}}\right.$
\end{tabular}


bioRxiv preprint doi: https://doi.org/10.1101/779793; this version posted April 18, 2020. The copyright holder for this preprint (which was not certified by peer review) is the author/funder, who has granted bioRxiv a license to display the preprint in perpetuity. It is made available under aCC-BY-NC-ND 4.0 International license.

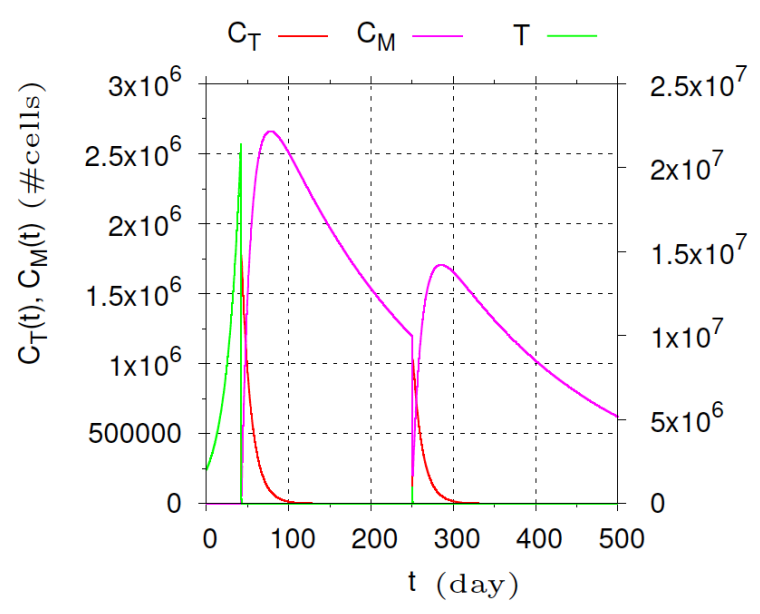

(a)

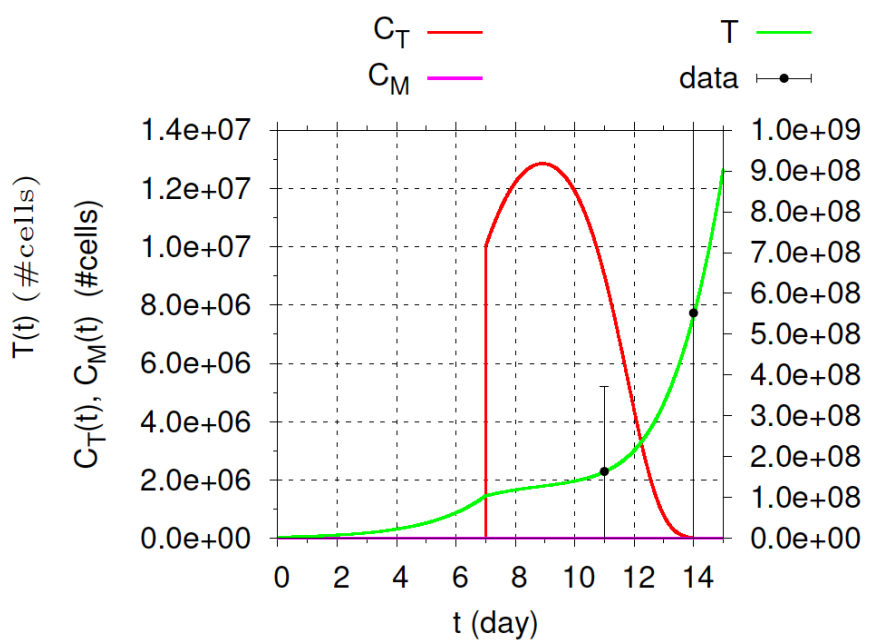

(b)

Fig. 2 Dynamics of $T, C_{T}$ and $C_{M}$ cell populations. (a) The immunotherapy with CAR T 123 on HDLM-2 and challenge are performed at $t=42$ and $t=250$ days, respectively. Tumor is rapidly eliminated after $C_{T}$ is introduced in the system. Soon after there is a decay of $C_{T}$, which is partially converted into $C_{M}$. Tumor remains undetectable until day 250 . Simulation is continued by carrying out a challenge at day 250. Upon contact with new tumor cells, $C_{M}$ is converted into $C_{T}$, which rapidly eliminates the tumor. Afterwards, immunological memory is partially recovered. (b) The immunotherapy with CAR T 19 on Raji-control is performed at day 7 . There is an expansion of effector $\mathrm{T}$ cells, which can reduce growth but not eliminate the tumor. Effector $\mathrm{T}$ cells are practically extinct at the end of the simulation. There is no formation of memory CAR $\mathrm{T}$ cells. Data extracted from [29]. 
bioRxiv preprint doi: https://doi.org/10.1101/779793; this version posted April 18, 2020. The copyright holder for this preprint (which was not certified by peer review) is the author/funder, who has granted bioRxiv a license to display the preprint in perpetuity. It is made available under aCC-BY-NC-ND 4.0 International license.

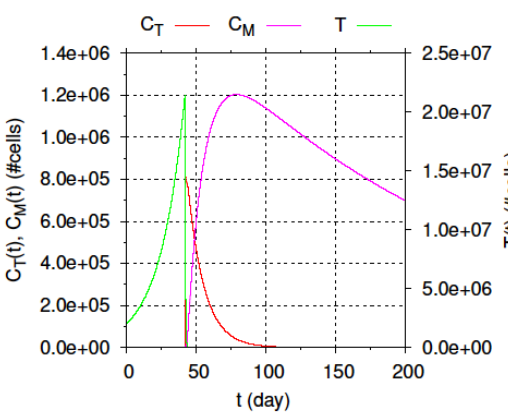

(a) CAR T dose: $1 \times 10^{6}$ cells Tumor elimination

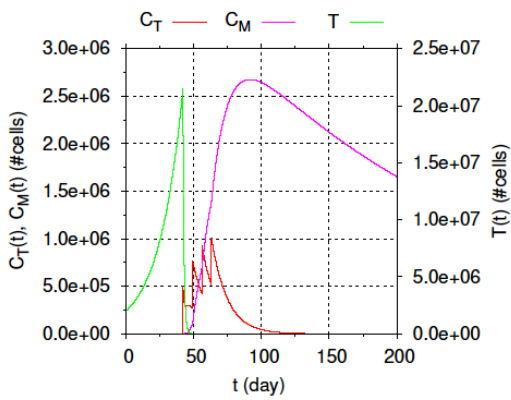

(d) 4 doses of $5 \times 10^{5}$ CAR $\mathrm{T}$ cells every 7 days

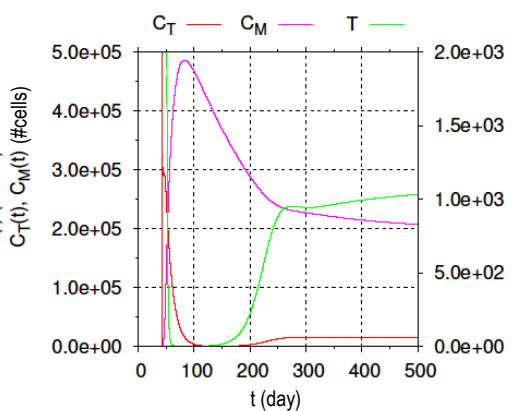

(b) CAR T dose: $5 \times 10^{5}$ cells Tumor dormancy

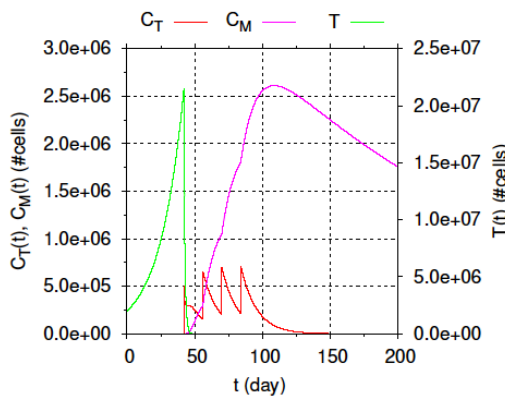
every 14 days

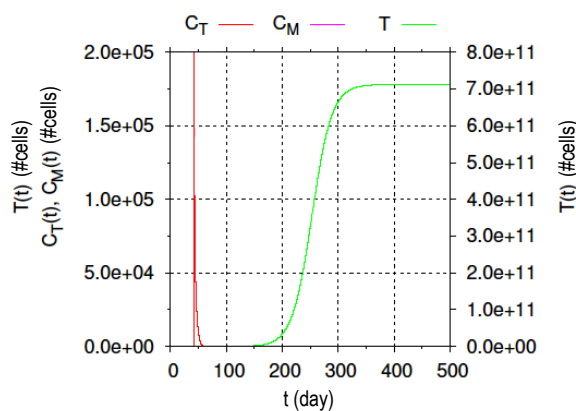

(c) CAR T dose with $2 \times 10^{5}$ cells Tumor escape

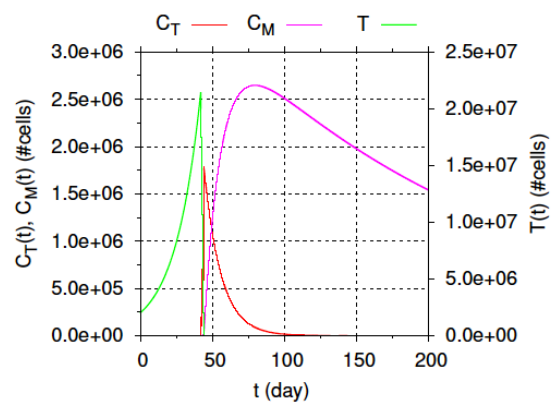

(f) 3 fractionated infusions over 3 days $(10 \%: \mathrm{d} 1,30 \%: \mathrm{d} 2$, and $60 \%: \mathrm{d} 3)$

Fig. 3 In silico prediction of the immunotherapy response to different CAR $\mathrm{T}$ cell dose. Initial HDLM-2 tumor burden amounts $2 \times 10^{6}$ cells. Top row: (a) with $1 \times 10^{6}$ CAR T cells injection, tumor elimination occurs around day 100; around $7 \times 10^{5}$ memory cells remain at $t=200$ days; (b) half of the previous CAR T cell dose $\left(5 \times 10^{5}\right)$ induces a strong decline in the tumor burden, together with a decrease in the number of CAR T cells. However, tumor rapidly resumes growth. After day 250 , the three cell populations slowly change towards an equilibrium state, in which a small pool of tumor cells coexists with the CAR T and memory T cell populations; (c) $2 \times 10^{5}$ CAR T cells dose is not able to control the tumor, which escapes and reaches the carrying capacity at day 350 . The fast decay of CAR T cells prevents the formation of a memory cell population. Bottom row: the total CAR T dose of $2 \times 10^{6}$ cells is fractionated into four equal portions and administered every (d) 7 days or (e) 14 days; (f) the dose is fractionated into 3 infusions of increasing dose values over 3 days. In all cases (d)-(f), the tumor is eliminated in a few days, followed by a decrease of the effector $\mathrm{T}$ cells. Fractionated infusions lead to the formation of memory $\mathrm{T}$ cells, although the quantity depends on the rest time between doses. 
bioRxiv preprint doi: https://doi.org/10.1101/779793; this version posted April 18, 2020. The copyright holder for this preprint (which was not certified by peer review) is the author/funder, who has granted bioRxiv a license to display the preprint in perpetuity. It is made available under aCC-BY-NC-ND 4.0 International license.

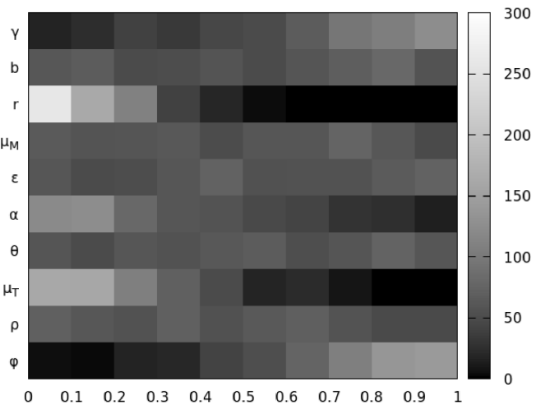

(a) Elimination (5\%)

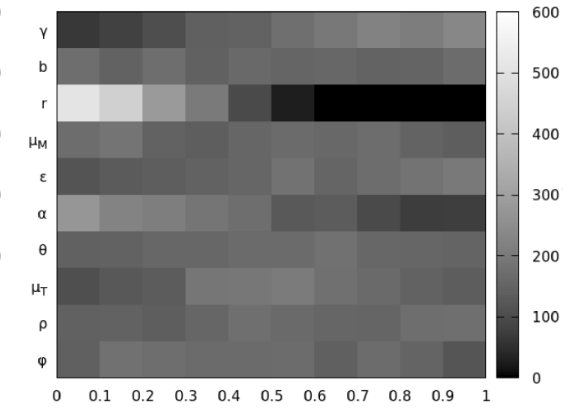

(b) Equilibrium (18\%)

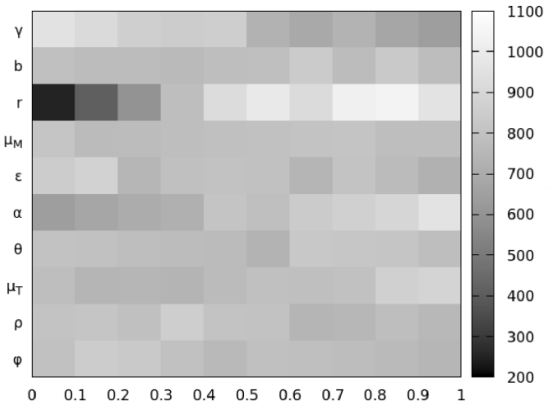

(c) Escape $(77 \%)$

Fig. 4 Frequency of occurrence of elimination (a), equilibrium (b), and escape (c) of the tumor for the scenario in which the initial HDLM-2 tumor burden is $2 \times 10^{6}$ cells and the immunotherapy with $2 \times 10^{6}$ cells CAR T is performed at day 42. Darker colors indicate lower frequency. All parameters are assumed to be uncertain, being uniform distributed random variables with range limited by $20 \%$ of the reference values indicated in the Supplementary Material, Table S6. Horizontal axes are associated with the normalized parameter values. We evaluated 10,000 cases by randomly sampling the parameter space. Tumor escape is more likely to occur, followed by the equilibrium and elimination. The respective percentage of these responses are indicated in parentheses.

(a)

$$
\begin{aligned}
& \mathrm{C}_{\mathrm{T}}-\quad \mathrm{T} \\
& \mathrm{C}_{\mathrm{M}}-\quad \text { data }
\end{aligned}
$$

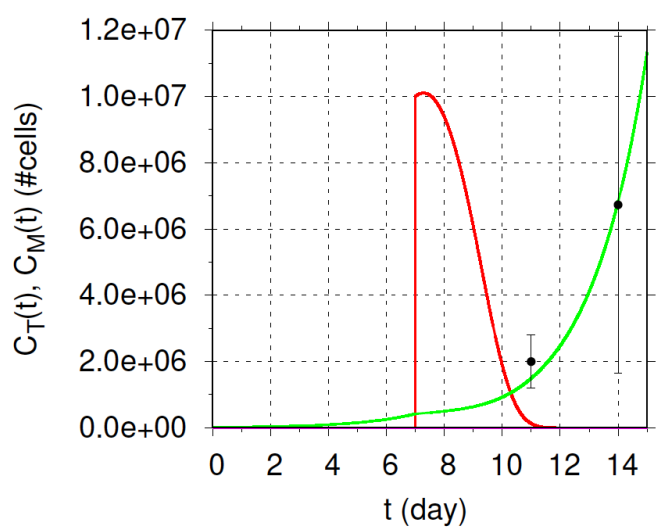

Raji-IDO + CAR T 19

$$
\alpha_{M L E}=1.46 \times 10^{-8}(\text { cell } \cdot \text { day })^{-1}
$$

(b) $3.0 \mathrm{e}+09$ $2.5 e+09$ $2.0 \mathrm{e}+09$

$1.5 \mathrm{e}+09$

$1.0 \mathrm{e}+09$

$5.0 \mathrm{e}+08$ $0.0 e+00$

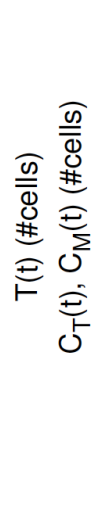

$$
\begin{aligned}
& \text { Raji-IDO + CAR T } 19+1-\mathrm{MT} \\
& \alpha_{M L E}=1.26 \times 10^{-8}(\text { cell } \cdot \text { day })^{-1}
\end{aligned}
$$

Fig. 5 System response to: (a) CAR T 19 immunotherapy; (b) CAR T 19 immunotherapy with IDO inhibitor (1-MT). Initial Raji-IDO tumor burden is $3 \times 10^{6}$ cells. At day $7,1 \times 10^{7}$ CAR T 19 cells were introduced and were able to reduce the velocity of the tumor growth at some extent. Such reduction was more significant when CAR T 19 therapy was combined with the IDO inhibitor (1-MT): the number of tumor cells were less than half of that without 1-MT at day 15. Model parameter $\alpha$ was estimated for these two cases, and their corresponding MLE values are indicated above. The parameter $\alpha$ was responsible to capture the effect of IDO inhibition and the 1-MT. Its value decreased for the Raji-IDO + CAR T 19 + 1-MT case, being small enough to promote a higher expansion of the CAR T cells, and ultimately leading to a more effective control on the tumor growth. However, both therapies were not able either to eliminate the tumor or build memory cells. Data extracted from 29 . 